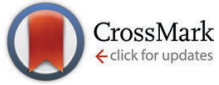

Cite this: Phys. Chem. Chem. Phys., 2014, 16, 22426

DOI: $10.1039 / c 4 c p 90133 a$

www.rsc.org/pccp

\section{Correction: A unified study for water adsorption on metals: meaningful models from structural motifs}

\author{
Guillem Revilla-López* and Núria López*
}

Correction for 'A unified study for water adsorption on metals: meaningful models from structural motifs' by Guillem Revilla-López et al., Phys. Chem. Chem. Phys., 2014, 16, 18933-18940.

Authors noticed erroneous values in Table 2 that should be the result of applying the formula: $\gamma_{\text {ads }}\left(\mathrm{eV} \AA^{-2}\right)=E_{\text {ads }} / A$ where $E_{\text {ads }}$ are the total adsorption energies for each motif, and $A$ the surface area where the motif was calculated.

Consequently the first paragraph of section 3.1, "Strain effects on DFT adsorption energies" from line 47 on page 18935 to line 8 on page 18936, should read:

The DFT-D2 adsorption energies for different sets of training structures under $\pm 4 \% d_{X Y}$ deformation are presented in Table 1 in eV per $\mathrm{H}_{2} \mathrm{O}$. Table 2 reports the same energies per surface area unit, $\AA^{-2}$, to better understand the experimental results. The direct comparison between data in Tables 1 and 2 indicates that the adsorption energies and surface energies follow a similar pattern. In both cases sqrt(37), 0.7 ML, shows the lowest energy. In contrast, ice-like bilayer, $0.67 \mathrm{ML}$, and Rosette, $0.5 \mathrm{ML}$, swap their position in the ranking with the latter being the least stable. Yet, in $\mathrm{Ru}(0001)$ dissociated ice-like bilayer has the lowest adsorption energy, $\gamma_{\text {ads }}$, followed by the other three in the same ordering.

Table 2 DFT-D2 calculated adsorption energies for H-down ice-like, sqrt(37) and Rosette structures on Pd, Pt and Ru. All energies are in eV $\AA^{-2}$. All $d_{X Y}$ are in plane deformations (in \%) with respect to the unstrained metal slab

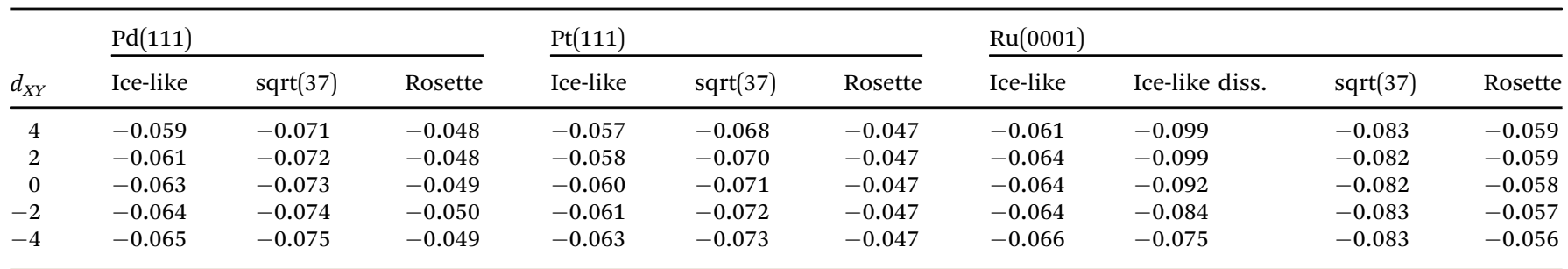

The Royal Society of Chemistry apologises for these errors and any consequent inconvenience to authors and readers. 